\title{
Enhanced Osteogenesis of Human Mesenchymal Stem Cells by Periodic Heat Shock in Self-Assembling Peptide Hydrogel
}

\author{
Jing Chen, MS, ${ }^{1}$ Zhong-Dong Shi, PhD, Xinying Ji, PhD, Jorge Morales, PhD, ${ }^{2}$ Jingwei Zhang, BS, \\ Navneet Kaur, MS, and Sihong Wang, $\mathrm{PhD}^{1}$
}

The mechanisms for the heat-induced osteogenesis are not completely known and the thermal regulation of human mesenchymal stem cell (hMSC) differentiation is not well studied. In this study, the direct effects of mild heat shock (HS) on the differentiation of hMSCs into osteoblasts in self-assembling peptide hydrogel and on tissue culture plates were investigated. hMSCs isolated from human bone marrow were seeded in conventional culture plates (two-dimensional [2D] culture) and on the surface of three-dimensional (3D) PuraMatrix peptide hydrogel (3D culture), followed by $1 \mathrm{~h} \mathrm{HS}$ at $41^{\circ} \mathrm{C}$ once a week during osteogenic differentiation. Alkaline phosphatase (ALP) activity was enhanced in both 2D and 3D cultures via periodic HS at early stage of differentiation; meanwhile, HS significantly increased the calcium deposition at day 19 and 27 of differentiation in both 2D and 3D cultures. The periodic HS also upregulated osteo-specific genes, osterix (OSX) on day 11, osteopontin $(O P)$ on day 19, and bone morphogenetic protein 2 (BMP2) on day 25 in 2D culture. In 3D PuraMatrix culture, the runt-related transcription factor 2 (Runx2) was upregulated by HS on day 25 of differentiation. The heat shock protein 70 (HSP70) was significantly upregulated by HS in differentiated hMSCs analyzed at $24 \mathrm{~h}$ after HS. These results demonstrate that HS induced an earlier differentiation of hMSCs and enhanced the maturation of osteoblasts differentiated from hMSCs. Therefore, mild HS treatment may be potentially used to enhance the bone regeneration using hMSCs. Our data will guide the design of in vivo heating protocols and enable further investigations in thermal treatments of MSC osteogenesis for bone tissue engineering.

\section{Introduction}

I N United States Alone, osteoarthritis (OA) affects about 70 million people and over 65 billion dollars are spent each year to treat the disease and related conditions. ${ }^{1}$ In clinical therapy for bone diseases, bone graft is often used to fill bone defects and promote bone regeneration. An autograft represents the current gold standard of bone graft; however, it is associated with problems, such as limited number of donors and donor-site trauma and morbidity. ${ }^{2}$ Allograft is used as an alternative, but also has inherent risks, including disease transmission, and altered biomechanical strength. ${ }^{3}$ Ceramic artificial bones can offer good strength, but they cannot provide an adequate environment for cell penetration and settlement deeply inside of the bone defects. ${ }^{4}$ In addition, the drawback of both is a lack of physiological remodeling that must take place over postsurgical years. ${ }^{5}$ Therefore, for functional bone tissue engineering, cells seeded with bioengineered scaffolds that are biodegradable, biocompatible, osteoconductive, and osteogenic are being investigated. ${ }^{6-10}$
Human mesenchymal stem cells (hMSCs) have the potential to differentiate into a variety of cell types, including osteoblasts, chondrocytes, and adipocytes, and they can also express key markers of endothelial cells and cardiomyocytes. ${ }^{2,11}$ Intensive studies have been done on influences of growth factors, ${ }^{12}$ cytokines, ${ }^{13}$ or mechanical loading ${ }^{14}$ on MSC differentiation into osteoblasts. However, the osteoblasts differentiated from MSCs are still not as functionally mature as primary adult cells. Further maturation and optimization of differentiation is needed. Temperature has an important impact on bone growth in vivo. It may be one of the missing factors in the regulation of the MSC differentiation.

Local diathermy can promote the bone repair after injury in vivo. Local hyperthermia $\left(38^{\circ} \mathrm{C}-41^{\circ} \mathrm{C}\right)$ treatment induced the new bone formation in rats following mechanical expansion at the sagittal suture. ${ }^{15}$ Hyperthermia is used as a thermotherapy for musculoskeletal diseases. It improves blood supply and, therefore, may influence bone metabolism and accelerate local bone formation. ${ }^{16}$ Microwave heating on

${ }^{1}$ Department of Biomedical Engineering and ${ }^{2}$ Electron Microscopy Center, The City College of the City University of New York, New York, New York. 
the joints of animal OA models inhibited the progress of cartilage damage. ${ }^{17}$ The localized heat that applied to articular joints likely involves the direct heating of chondrocytes, osteoblasts, and osteoprogenitor cells. Early studies have indicated that heat $\left(1.5^{\circ} \mathrm{C}-3^{\circ} \mathrm{C}\right.$ above regular body temperature) stimulates bone growth and embryonic development of the animals. ${ }^{18,19}$ In addition, local temperature rise at the bone fracture site was observed to be closely related with new bone formation. ${ }^{20}$ However, very few studies have been conducted to investigate thermal effects on bone marrow stem cell differentiation into osteoblasts, and their results in terms of alkaline phosphatase (ALP) activities and calcium deposition varied from time to time during differentiation. ${ }^{21}$ In another study, conditioned media from heat shock-treated human fetal osteoblast cells were able to promote the osteogenesis of rabbit MSCs. ${ }^{22}$ Most of the previous studies focused on the effects on cell lines, such as the telomeraseimmortalized hMSC line (hMSC-TERT) ${ }^{23}$ or osteosarcomaderived cell lines. ${ }^{24}$ All the previous studies were performed using two-dimensional (2D) culture configurations. The exact mechanism of thermal-induced bone growth is unclear. Whether thermal regulation plays a role in adult stem cell differentiation is also not well studied, especially in a threedimensional (3D) culture environment.

In this study of hMSC osteogenesis under the thermal stimulation, synthetic peptide hydrogel, 0.25\% PuraMatrix with storage modulus of $2.5 \mathrm{kPa}^{25}$ at $0.5 \%$, and conventional 2D tissue culture plates with Young's modulus of $3.5 \mathrm{GPa}^{26}$ were chosen as 3D culture matrix and 2D culture substrates, respectively. These two substrates would better mimic the mechanical environment that bone marrow MSCs experience in vivo while they migrate from the bone marrow niche (storage modulus of $0.2 \mathrm{kPa}^{27}$ ) and differentiate into osteoblasts for bone (Young's modulus of $9 \mathrm{GPa}^{26}$ ) repair. The peptide of PuraMatrix consists of a 16-amino acid sequence (RAD16-I, AcN-RADARADARADARADA-CONH ${ }_{2}$ ) and can self-assemble in the presence of cations in physiological solutions to form a 3D interweaving nanofiber scaffold containing over $99 \%$ of water. ${ }^{28}$ The fiber diameter and pore sizes in the hydrogel are about $10 \mathrm{~nm}$ and $50-200 \mathrm{~nm}$, respectively. PuraMatrix promotes cell attachment and migration across a number of cell types. ${ }^{29,30}$ Cellular phenotypes have been studied in PuraMatrix culture using hepatocytes, $^{31,32}$ chondrocytes, ${ }^{33}$ osteoblasts, ${ }^{34}$ and MSCs. ${ }^{35,36}$ In addition, the osteoconductive ability of PuraMatrix was investigated on bone regeneration in a mouse calvaria defect model. ${ }^{4}$ It was also shown to be a potential biomaterial to fill in the bone defect through injection in vivo. ${ }^{37}$

Our preliminary study using only one heating cycle did not show significant heat shock (HS)-enhancement on osteogenesis. Therefore, periodic HS was used to have prolonged thermal effects on the MSC differentiation during 4 weeks. Considering the elevated body temperature $\left(\sim 39^{\circ} \mathrm{C}\right)$ during exercise ${ }^{38}$ and knowing a common recommendation to OA patients from the Arthritis Foundation is to take a daily hot shower, once-a-week heating at $41^{\circ} \mathrm{C}$ was chosen in this study to provide enough heat stimulation. Osteogenesis was measured by ALP activities, quantitative calcium deposition, and gene expressions of osteogenic markers. Results of this study would validate a suitable heating protocol for MSC osteogenesis, and thus benefit further investigations of using thermal treatments for bone regeneration in vivo.

\section{Materials and Methods}

All reagents and chemicals without manufacture labels were purchased from Sigma-Aldrich (St. Louis, MO). hMSCs were isolated from human bone marrow, and then characterized by FACS analysis. Cells were seeded in 2D culture plates or 3D PuraMatrix gel, and exposed to $1 \mathrm{~h} \mathrm{HS}$ at $41^{\circ} \mathrm{C}$ once a week during osteogenic differentiation. Cell morphology and cell-cell as well as cell-matrix interactions on the hydrogel scaffold were analyzed by scanning electron microscopy (SEM), and calcium deposits from the cells were localized by calcium X-ray spectrum. The analysis of induction of osteogenic differentiation and the extent of mineralization was also performed using quantitative ALP and calcium assays, respectively. The gene expression of the osteo-specific markers was measured by real-time polymerase chain reaction (PCR) and heat shock proteins (HSP27, HSP70, and HSP90) expression was further determined by Western blot analysis.

\section{Isolation and culture of bone marrow hMSCs}

Human bone marrow (BM) from the iliac crest of a 27year-old donor was purchased (AllCells LLC, Berkeley, CA). Mononuclear cells were enriched and retrieved with RosetteSep MSC enrichment cocktail (StemCell Technologies, Vancouver, Canada) according to the manufacturer's instructions, and then cultured in tissue culture flasks with the MSC growth medium (MSCGM) consisting of the Dulbecco's modified Eagle's medium-low glucose, $10 \%$ fetal bovine serum (Atlanta Biologicals, Lawrenceville, GA), and 1\% penicillin-streptomycin (Invitrogen, Carlsbad, CA) in an incubator at $37^{\circ} \mathrm{C}$ with $5 \% \mathrm{CO}_{2}$. Subculture was performed at a density of 5000 cells $/ \mathrm{cm}^{2}$. Passage 4 MSCs were used in this study.

\section{Characterization of hMSCs by surface markers and flow cytometry}

Isolated hMSCs at the concentration of $10^{6}$ cells $/ \mathrm{mL}$ in MSCGM were incubated with mouse anti-human antibodies (BD Biosciences, San Jose, CA). Samples were either double stained with a pair of antibodies, such as the negative control pair of IgG-phycoerythrin (PE) \& IgG-fluorescein isothiocyanate (FITC), CD45-FITC\&CD44-PE, CD147-FITC\&CD29PE, CD147-FITC\&CD34-PE, or single stained with CD146-PE for $20 \mathrm{~min}$ at room temperature. The cells were analyzed on a FACSCalibur flow cytometer (BD Biosciences) with adjusted fluorescence compensation setting and processed on the same machine. Negative control samples were used to set up the thresholds of quadrant markers.

\section{Assembling of peptide hydrogel and cell seeding}

PuraMatrix (BD Biosciences) was assembled using MSCGM following the manufacturer's protocol in 24-well plates, $300 \mu \mathrm{L}$ of $0.25 \%(\mathrm{w} / \mathrm{v})$ gel per well. Cell seeding densities were $10^{4} \mathrm{hMSC}$ per well for $2 \mathrm{D}$ culture and $4 \times 10^{4}$ hMSCs per well for 3D PuraMatrix culture using surface seeding. Osteogenic differentiation was induced with the osteogenic medium the day (day 0) after seeding and was used for control cultures. The osteogenic medium consists of MSCGM supplemented with $50 \mu \mathrm{M}$ ascorbic acid phosphate (AsAP) (Wako Chemicals USA, Richmond, VA), $0.1 \mu \mathrm{M}$ 
dexamethasone, and $10 \mathrm{mM} \beta$-glycerol phosphate. The medium was changed every 3-4 days.

\section{Heat exposure}

hMSCs in osteogenic (OS) cultures were exposed to mild HS periodically on day $3,10,17$, and 24 . The transient $1 \mathrm{~h}$ heating at $41^{\circ} \mathrm{C}$ was performed using a cell culture incubator precalibrated with an accuracy of $\pm 0.2^{\circ} \mathrm{C}$. Choosing $41^{\circ} \mathrm{C}$ as the heating temperature was based on a previous study that repeated 1-h exposure to $41^{\circ} \mathrm{C}$ once every 3 days generated highest ALP activities and calcium deposition in hMSC 2D culture compared to $39^{\circ} \mathrm{C}$ or $42.5^{\circ} \mathrm{C} .^{21}$ The medium was changed after heating and the cells were back to the $37^{\circ} \mathrm{C}$ incubator. The control samples stayed in the $37^{\circ} \mathrm{C}$ incubator while the medium was changed at the same time as the heat shocked samples.

\section{Differentiation and visualization of minerals}

The morphology changes of hMSCs in different culture conditions were observed by phase microscopy using a Zeiss Axio Observer Z1 Inverted microscope. Von Kossa staining was used to visualize mineralization on day $24 .{ }^{39}$ Briefly, hMSCs were rinsed with the Tyrode's balanced salt solution, fixed with 10\% buffered formalin (Fisher Scientific, Pittsburgh, PA) for $30 \mathrm{~min}$, incubated with $2 \%$ silver nitrate solution for $10 \mathrm{~min}$ in the dark, rinsed with $\mathrm{ddH}_{2} \mathrm{O}$, and exposed to light for $15 \mathrm{~min}$. Bright-field images of stained samples were captured with a Zeiss Axiovert 40 CFL inverted microscope.

\section{Surface morphology of 3D culture and calcium localization}

To study the detailed surface morphology of hMSCs on PuraMatrix, SEM was used. Lab-Tek chambered coverglass (Thermo Fisher, Rochester, NY) was used to culture hMSCs $\left(4 \times 10^{4}\right.$ cells $/$ well $)$ on $0.25 \%$ PuraMatrix for SEM analysis. On day 26, samples were fixed in $4 \%$ glutaraldehyde (Electron Microscopy Sciences, Hatfield, PA) overnight at $4^{\circ} \mathrm{C}$, followed by ethanol dehydration, and critical point drying. Samples were then sputter-coated with $10 \mathrm{~nm}$ of goldpalladium or carbon coated with a carbon thread evaporator. The gold-coated samples were examined under Supra 55 VP (Carl Zeiss MicroImaging, Thornwood, NY) using the in-lens detector at $5 \mathrm{kV}$ to collect secondary electron (SE) images. ${ }^{40}$ The carbon-coated samples were analyzed by energy dispersive X-ray spectrometry (EDS) at $15 \mathrm{kV}$ using the Apollo 40 EDAX detector to generate calcium $X$-ray spectrum.

\section{Quantitative ALP assay}

Samples were lysed with $300 \mu \mathrm{L}$ of ALP lysis buffer, $0.5 \%$ Triton X-100 (Bio-Rad Laboratories, Hercules, CA), on day 6 and 12 during differentiation. The hydrogel samples were homogenized with pipetting and sonicated for $10 \mathrm{~min}$. The samples were vortexed and incubated with an alkaline buffer solution and a phosphatase substrate solution at $37^{\circ} \mathrm{C}$ for $15 \mathrm{~min} .{ }^{41}$ The ALP activity in $\mathrm{nmol} / \mathrm{min}$ was calculated by comparing samples' absorbance of p-nitrophenol product at $405 \mathrm{~nm}$ with that of p-nitrophenol standards using the SpectraMax M2e microplate reader (Molecular Devices, Silicon Valley, CA). The statistical comparison was drawn between heat shocked and nonheat shocked osteogenic samples.

\section{Calcium quantification}

Samples for calcium deposition assay were collected on day 19 and 27 during differentiation using $0.5 \mathrm{~N} \mathrm{HCl}$. Calcium was extracted from the cells by shaking the samples on an orbital shaker for $4 \mathrm{~h}$ at $4^{\circ} \mathrm{C}$, followed by centrifugation at $500 \mathrm{~g}$ for $2 \mathrm{~min}$. The supernatant was collected for calcium determination according to instructions provided in the Calcium Liquicolor kit (Stanbio Laboratory, Boerne, TX). Absorbance was read at $550 \mathrm{~nm}$. The total calcium amount in $\mu \mathrm{g} /$ well was calculated by comparing to the standard curve.

\section{Gene expression measured by real-time reverse transcription-polymerase chain reaction}

Total RNA was extracted from hMSC samples on day 11, 19, and 25 during osteogenesis using the TRIzol Reagent (Invitrogen) and the RNeasy Mini kit (Qiagen, Valencia, CA) following the manufacturer's instructions, and used to synthesize cDNA with the Cells-to-cDNA II kit (Ambion, Austin, TX), followed by real-time PCR analysis in ABI Prism 7000 Sequence Detection System (Applied Biosystems, Foster City, CA). Table 1 includes reverse transcription (RT)-PCR primers of osteogenic genes, the bone morphogenetic protein $2(B M P 2)$, osteopontin $(O P)$, the runt-related transcription

Table 1. Primer Sequences for Real-Time Reverse Transcription-Polymerase Chain Reaction

\begin{tabular}{|c|c|c|}
\hline Gene & $5^{\prime}$ to $3^{\prime}$ sequence & $\begin{array}{l}\text { Amplicon } \\
\text { size (bp) }\end{array}$ \\
\hline \multirow[t]{2}{*}{ BMP2 } & Forward: CATGCCATTGTTCAGACG & 172 \\
\hline & Reverse: TGTACTAGCGACACCCACA & \\
\hline \multirow[t]{2}{*}{ Osteopontin } & Forward: ACCCTTCCAAGTAAGTCC & 349 \\
\hline & Reverse: TGTCCTCGTCTGTAGCAT & \\
\hline \multirow[t]{2}{*}{ Runx2 } & Forward: AAATCGCCAGGCTTCATA & 447 \\
\hline & Reverse: CTGCCAGGAGTGGTCAAA & \\
\hline \multirow[t]{2}{*}{ Osterix } & Forward: CCTGCGACTGCCCTAATT & 123 \\
\hline & Reverse: GCGAAGCCTTGCCATACA & \\
\hline \multirow[t]{2}{*}{ GAPDH } & Forward: GGATTTGGTCGTATTGGG & 205 \\
\hline & Reverse: GGAAGATGGTGATGGGATT & \\
\hline
\end{tabular}

BMP2, bone morphogenetic protein 2; Runx2, runt-related transcription factor 2; GAPDH, glyceraldehyde-3-phosphate dehydrogenase. 
factor 2 (Runx2), and osterix (OSX) ordered from Integrated DNA Technologies (Coralville, IA). Glyceraldehyde-3-phosphate dehydrogenase (GAPDH) was used as an internal control. The thermal profile for all reactions was $95^{\circ} \mathrm{C}$ for $10 \mathrm{~min}$, followed by 45 cycles at $95^{\circ} \mathrm{C}$ for $15 \mathrm{~s}, 55^{\circ} \mathrm{C}$ for $35 \mathrm{~s}$, and $72^{\circ} \mathrm{C}$ for $35 \mathrm{~s}$. Relative expression levels in heat shocked or nonheat shocked osteogenic samples were calculated as an average of the ratio, to the value of that of undifferentiated cells for a specific gene on individual days after normalization with GAPDH.

\section{HSP detection by Western blot and ELISA}

Samples were lysed at $24 \mathrm{~h}$ after HS on day $4,11,18$, and 25 during differentiation. Cells in PuraMatrix culture were extracted first from a gel following the manufacturer's instruction before cell lysis. The lysis buffer for 3D culture contains $1 \%$ sodium dodecyl sulfate (SDS), $50 \mathrm{mM}$ Tris- $\mathrm{HCl}$, $5 \mathrm{mM}$ EDTA, and protease inhibitors, while 2D culture samples were lysed with $1 \times$ extraction buffer of HSP70 EIA kit (Assay Designs, Ann Arbor, MI) supplemented with protease inhibitors. Cell lysate of 3D culture was briefly sonicated. The cell lysate was then centrifuged at 21,000 $\mathrm{g}$ for $10 \mathrm{~min}$ at $4^{\circ} \mathrm{C}$ and the supernatant was collected for Western blot or ELISA. The total protein concentration was determined by the Bio-Rad protein assay kit. 10\% Tris- $\mathrm{HCl}$ Ready Gel and polyvinylidene fluoride membranes (Bio-Rad Laboratories) were used. The primary antibodies were monoclonal mouse anti-human HSP27 (Assay Designs), HSC70\&HSP70, HSP90 (Santa Cruz Biotechnology, Santa Cruz, CA), and actin (Millipore, Billerica, MA). The secondary antibody was horseradish peroxidase-conjugated goat anti-mouse IgG (Assay Designs). The Tetramethylbenzidine substrate kit (Vector Laboratories, Burlingame, CA) was used to visualize the protein bands. Membranes were dried and scanned into digital images. Protein bands were analyzed quantitatively using Quantity One software (Bio-Rad Laboratories). The HSP expression was presented as a ratio of total intensity of HSPs normalized by that of actin after global background subtraction to equalize the total protein loading for each sample.

The concentrated protein samples of 2D culture were also used for inducible HSP70 measurement. The 3D protein samples collected were buffer-exchanged using both Amicon Ultra-4 and Ultra-0.5 Centrifugal Filter columns (Millipore) so that SDS concentrations in the final lysate would be compatible with the commercial HSP70 EIA kit. HSP70 ELISA was performed following the manufacturer's instruction. HSP70 expression in $\mathrm{ng} / \mathrm{mg}$ was presented as a ratio of the HSP70 concentration normalized by the total protein concentration of the same sample.

\section{Statistical analysis}

All values are expressed as mean \pm standard deviation (SD) and analyzed statistically using a two-tailed Student's $t$-test. The level of significance was set at $p<0.05$.

\section{Results}

\section{Expression of surface markers in isolated hMSCs}

In Figure 1, flow cytometric analysis shows that passage 4 of isolated hMSCs was over $80 \%$ positive for surface markers of CD44 (hyaluronan receptor), CD29 (integrin $\beta 1$ ), and CD147 (extracellular matrix [ECM] metalloproteinase inducer), slightly positive for CD146 (melanoma cell adhesion molecule), and more than 90\% negative for CD45 (leukocyte common antigen) and CD34 (lipopolysaccharide receptor). CD34 and CD45 are surface markers of the hematopoietic lineage, and CD146 is the surface marker of the endothelial cell lineage, an epitope suggested as a biomarker for MSCs.

\section{Morphological changes during differentiation of hMSCs}

hMSCs underwent a dramatic morphology change with the osteogenic (OS) medium, while obvious cell proliferation was only observed in undifferentiated (Ctrl) hMSCs. Figure 2 shows phase-contrast images of different culture conditions on day 7, 14, and 21 during differentiation. In 2D Ctrl culture, hMSCs were spindle-shaped with better alignment when cell numbers increased. For 3D Ctrl culture on $0.25 \%$ PuraMatrix, hMSCs remained in fibroblast-like morphology and migrated inside PuraMatrix. In OS 2D culture, abundant secretions of ECM and small nodular aggregates were detected on day 14 and 21, respectively. In contrast, small cell aggregates were formed in OS 3D PuraMatrix culture on day 7 , and their sizes increased in day 14 and 21 cultures along with dark regions of mineral confirmed by von Kossa staining of mineral deposits on day 24 shown as black nodules in Figure 3. No mineral was detected in 2D Ctrl culture. However, in 3D Ctrl culture, there was mineral staining, but much less intensive compared with OS 3D culture.

\section{Surface morphology of hMSCs on PuraMatrix and localization of calcium deposition}

Figure 4 includes SEM images revealing detailed surface morphology of hMSCs on PuraMatrix and cell-hydrogel interactions. Figure 4A and B are SEM images from goldcoated samples. Undifferentiated hMSCs remained as fibroblast-like (Fig. 4A), while differentiated hMSCs formed aggregates (Fig. 4B). The network composed of cell protrusions into PuraMatrix nanofibers was observed in both growth and differentiation conditions. The inset picture in Figure 4C is an SEM image of a carbon-coated hMSC sample in the growth condition. Calcium X-ray spectrum of the same specimen showed that there was no significant amount of calcium deposition in the hMSC growth culture on PuraMatrix (Fig. 4C). As a contrast, mineralization sites were largely detected and distributed in and around differentiated hMSCs with variant density and calcium intensity shown by the calcium X-ray intensity map (Fig. 4D). The inset picture in Figure $4 \mathrm{E}$ is the SEM image of the same specimen used to obtain Figure 4D. In particular, a crystal structure was located under a high magnification of SEM shown in Figure 4F, which is a zoom-in SEM image of areas around the small red circle in the inset of Figure 4E. The high peak of calcium at the corresponding location in the calcium X-ray spectrum (Fig. 4E) confirmed that the crystal structure indicated by the red circle in Figure 4F was made of calcium.

\section{ALP activity}

ALP activity is one marker of early stage osteogenic (OS) differentiation. The significant increase of ALP activities by 

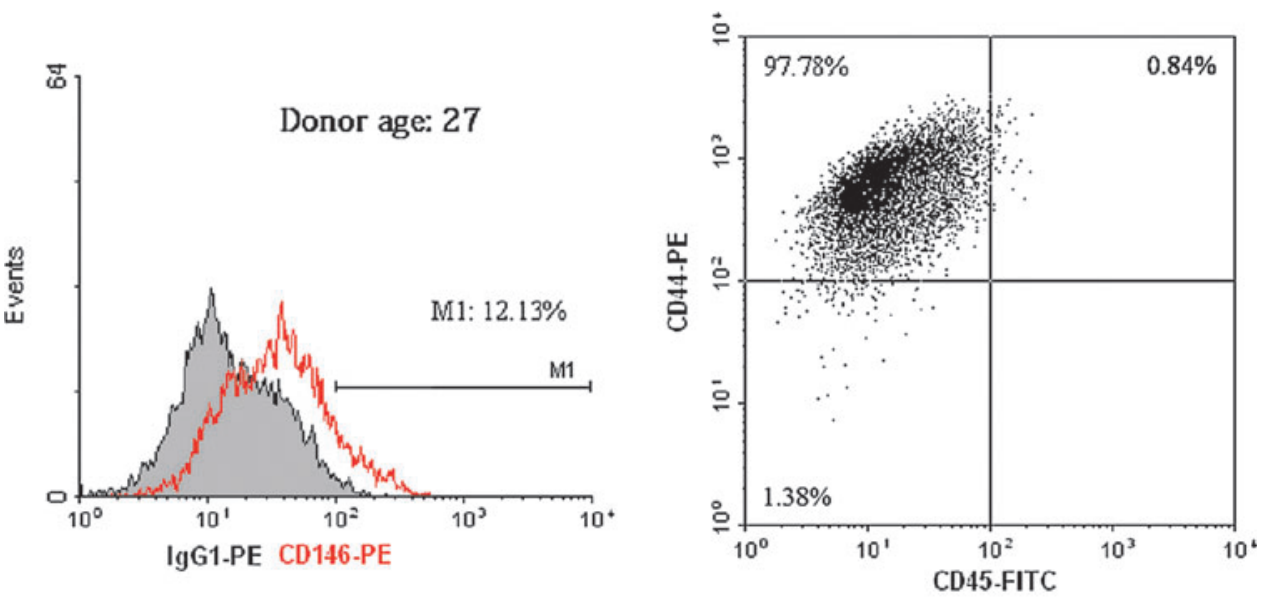

FIG. 1. Characterization of human mesenchymal stem cells (hMSCs) by flow cytometric analysis. Isolated hMSCs were positive to surface markers CD146, CD44, CD29, and CD147, and negative to CD45 and CD34. Color images available online at www.liebertpub.com/tea

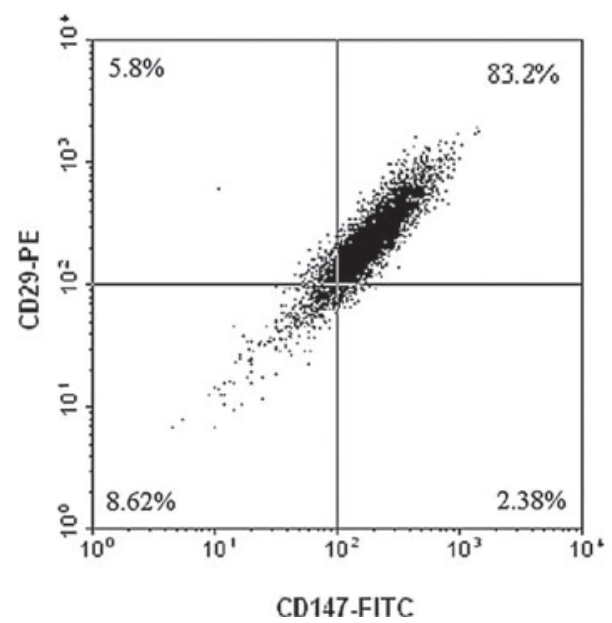

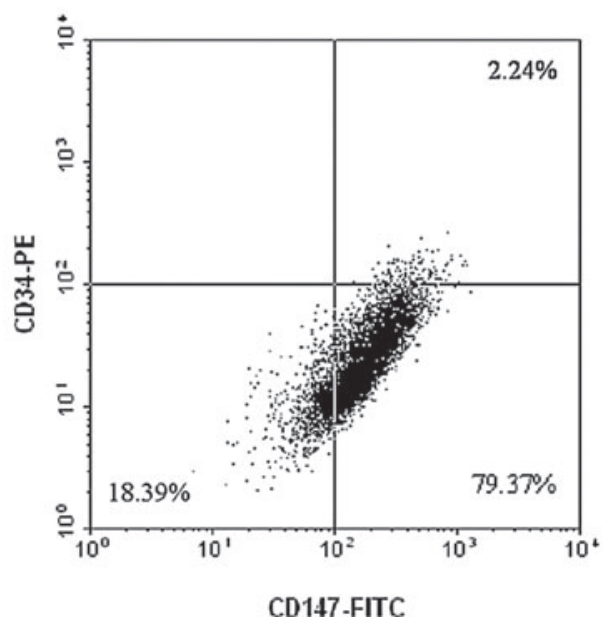

HS was observed on day 6 in 2D OS culture, while there was no significant ALP increase comparing between HS and nonHS 2D OS conditions on day 12 (Fig. 5A). In 3D PuraMatrix culture, the HS effect was less significant on the increase of ALP activities on day 6. Interestingly, HS significantly reduced ALP activities on day 12 in 3D OS culture (Fig. 5B). The $1-\mathrm{h}$ heat exposure at $41^{\circ} \mathrm{C}$ resulted in a 1.24-fold increase of ALP activities in 2D OS culture and 1.35-fold in 3D OS hydrogel culture on day 6 .

\section{Enhanced mineralization by HS}

Calcium deposition was used as an indicator of late stage osteogenesis and maturation of osteoblasts differentiated from MSCs. As shown in Figure 6, periodic HS at $41^{\circ} \mathrm{C}$ significantly enhanced the mineralization in 2D OS culture on day 19 and 3D OS PuraMatrix culture on day 19 and 27, while the enhancement effect of HS was less remarkable in 2D OS culture on day 27. Calcium deposition was increased by 1.64-fold and 1.49-fold in heat shocked osteogenic samples compared to nonheat shocked osteogenic ones in 2D culture and 3D culture, respectively, on day 19 and 1.34-fold in 3D culture on day 27.

\section{Dynamic expression of osteo-specific genes}

Gene expression of osteogenic lineage was assessed by realtime RT-PCR on day 11, 19, and 25 during differentiation.
Figure 7 shows that in 2D culture, osteogenic genes $B M P 2$, $O P, \operatorname{Runx} 2$, OSX were all upregulated in osteogenic samples compared to $2 \mathrm{D}$ control samples at growth conditions. Furthermore, the expression levels of these osteo-specific genes were increased in the late differentiation stage compared with the early stage, which indicated the progression of MSC osteogenesis in 2D culture. HS further enhanced the expression of OSX on day 11, OP on day 19, BMP2 and Runx2 on day 25. BMP2 and Runx2 were downregulated by HS in 2D culture on both day 11 and day 19. Figure 8 shows the real-time RT-PCR results of four genes in 3D PuraMatrix culture. BMP2, Runx2, and $O P$ had the similar gene expression patterns as that of 2D culture under the periodic thermal stimulation. However, the OSX gene had an opposite expression pattern under thermal stimulation in 3D PuraMatrix culture compared to the 2D culture. Interestingly, for 3D PuraMatrix culture, the osteogenic medium did not induce the upregulation of four genes except for Runx2.

\section{Expression of HSPs}

The expression of HSPs (HSP27, HSC70+HSP70, and HSP90) was investigated at $24 \mathrm{~h}$ after HS on day 4, 11, 18, and 25 during differentiation. Table 2 lists the HSP expression data quantified from the Western blot membranes. It shows that HSP27 expression was significantly upregulated 

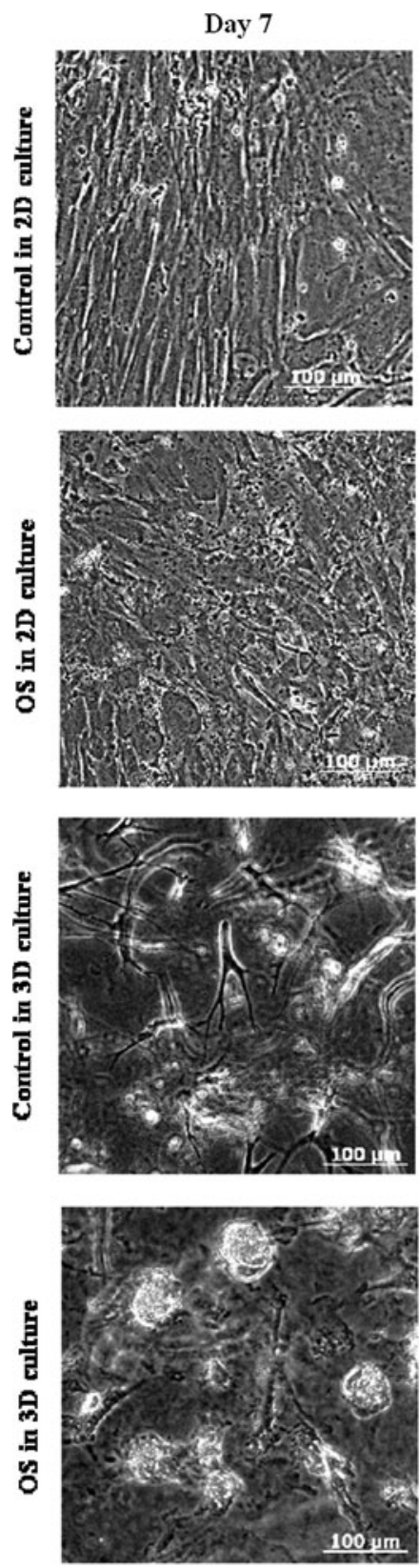

Day 14
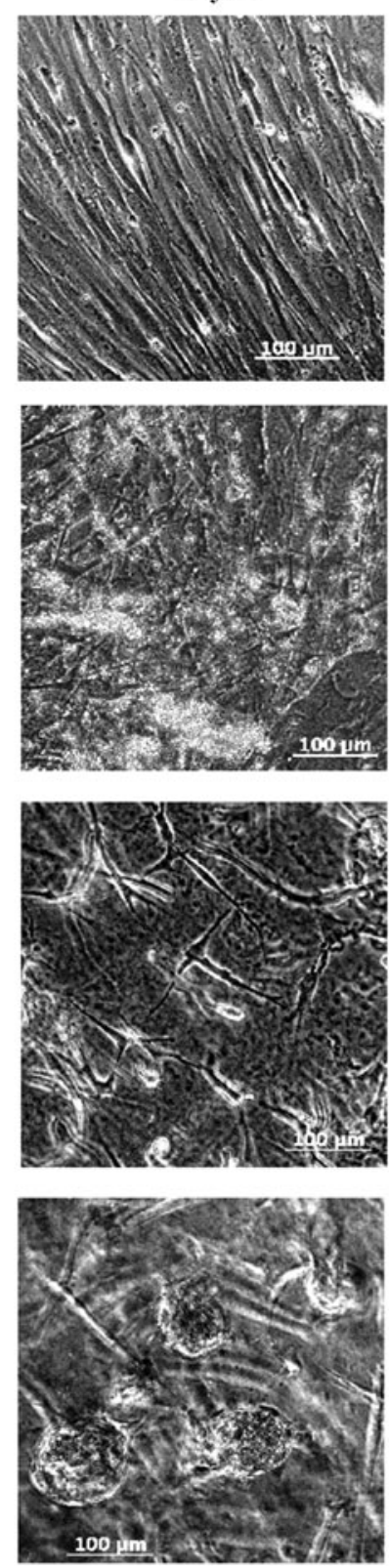

Day 21
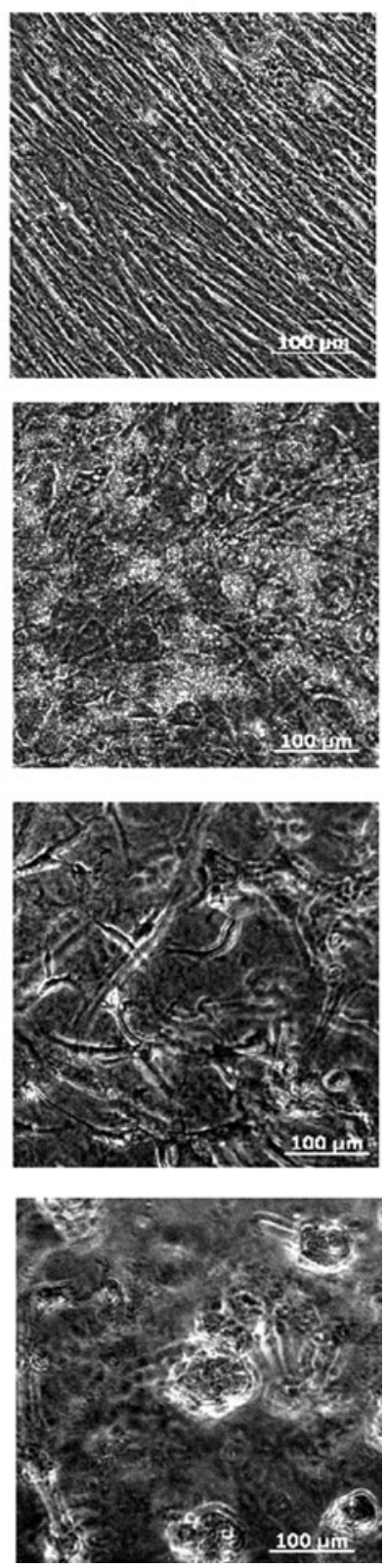

FIG. 2. Phase-contrast images showing the morphology of hMSCs cultured on $0.25 \%$ PuraMatrix ${ }^{\mathrm{TM}}$ or in twodimensional (2D) culture plates in osteogenic or growth medium on day 7,14 , and 21 during differentiation. in osteogenic (OS) samples compared with undifferentiated control (Ctrl) samples, while there was no significant increase of HSP27 in heat shocked osteogenic (HS) samples compared with OS samples in both 2D and 3D cultures. In contrast, the expression of HSC70 + HSP70 was significantly downregulated in OS samples compared with Ctrl samples, but HS significantly increased its expression in HS samples compared to OS samples in 2D culture. For HSP90 expression, upregulation was observed in OS samples compared to Ctrl samples and downregulation in HS samples compared with OS samples in 2D culture with the only significance on day 4. In general, the HSP expression levels in 3D PuraMatrix culture were lower compared with 2D culture, and too low to be detected using Western blot except for HSP27 on day 11 and beyond. The expression patterns of individual HSPs in different conditions (Ctrl, OS, HS) on one sample collection day and their corresponding Western blot membranes were displayed in Figure 9. In 2D culture, HSP27 expression was much higher than HSC70 + HSP70 or HSP90. The expression pattern of inducible HSP70 measured by ELISA was similar to that of HSC70+HSP70 (constitutive + inducible), and inducible HSP70 expression in 3D culture was also significantly lower compared with 2D culture shown in Figure 10.

\section{Conclusion and Discussion}

In this study, effects of periodic $\mathrm{HS}\left(41^{\circ} \mathrm{C}\right.$ once a week for $1 \mathrm{~h}$ ) on hMSC osteogenic differentiation were investigated in both 2D conventional culture and 3D PuraMatrix (peptide hydrogel) culture. In-house isolated hMSCs in the osteogenic medium formed mineralized aggregates in 2D culture plates and 3D peptide hydrogels (Figs. 2 and 3). Largely distributed calcium deposits were detected in differentiated hMSCs on 

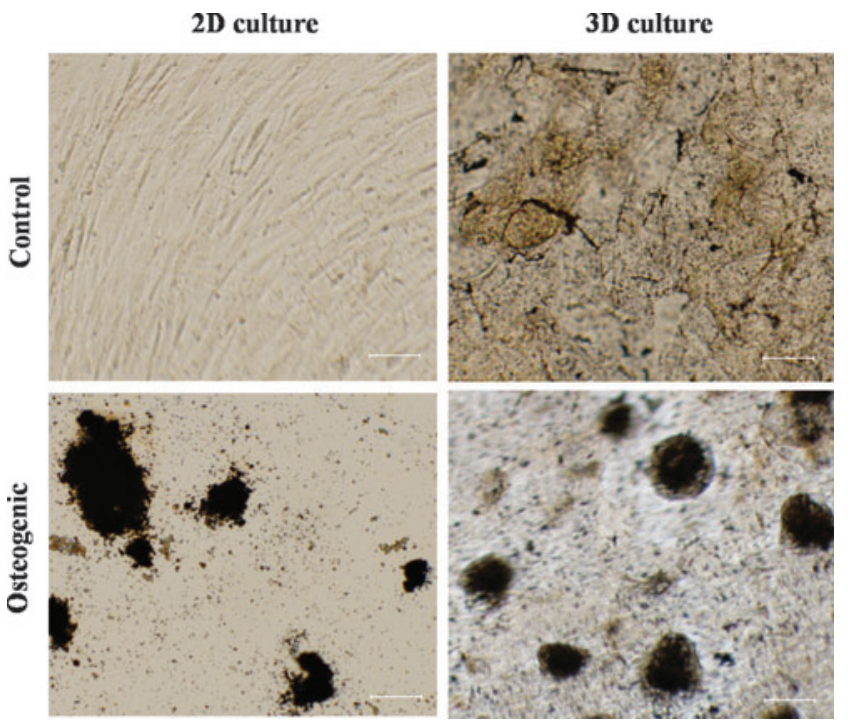

FIG. 3. Von Kossa staining for mineral deposits from hMSCs cultured in 2D plates and on three-dimensional (3D) PuraMatrix in the normal growth condition and osteogenic condition at day 24 after differentiation. Scale bar $=50 \mu \mathrm{m}$. Color images available online at www.liebertpub.com/tea
PuraMatrix (Fig. 4). Meanwhile, mild HS was able to significantly facilitate the early osteogenic differentiation indicated by the ALP activity (Fig. 5) and enhance the maturity of differentiated osteoblasts in the late stage shown by the calcium deposition in both 2D and 3D cultures (Fig. 6). In addition, thermal effects on osteo-specific genes were highly dynamic with a pattern of downregulated gene expression of $B M P 2, \operatorname{Run} x 2$, and $O P$ in the early stage of osteogenic differentiation, but upregulation of these genes in the late stage in both 2D and 3D cultures. Overall, our results demonstrate that periodic mild HS can be a potential simple approach to enhance hMSC differentiation into osteoblasts and promote earlier differentiation, and thus may have a significant impact on future in vivo applications.

Our results of enhanced ALP activities in the early osteogenic differentiation and calcium deposition by periodic HS at $41^{\circ} \mathrm{C}$ for $1 \mathrm{~h}$ in both $2 \mathrm{D}$ and $3 \mathrm{D}$ cultures are consistent with an early study of HS effects on hMSC osteogenic differentiation in $2 \mathrm{D}$ culture. ${ }^{21}$ In that study, Shui et al. showed that both ALP activities and calcium deposition in differentiated hMSCs linearly increased with temperatures ranging from $33^{\circ} \mathrm{C}$ to $41^{\circ} \mathrm{C}$ after multiple 1 -h heat exposure once every 3 days up to 21 days. HS seems to speed up the differentiation process by shifting the peak of ALP activities much earlier
FIG. 4. The morphology of (A) undifferentiated hMSCs and (B) differentiated hMSCs on PuraMatrix analyzed by scanning electron microscopy (SEM); (C) Calcium X-ray spectrum of hMSCs on PuraMatrix in the growth condition with an inset SEM image of the same sample; (D) Calcium X-ray map of heat-shocked \& differentiated hMSCs on PuraMatrix with purple areas indicating calcium locations; (E) Calcium X-ray spectrum of a specific spot in its SEM inset image circled in red color; (F) A zoom-in SEM image showing a calcium crystal indicated by a red circle, the same spot highlighted in the SEM inset in (E). All scale bars are $20 \mu \mathrm{m}$ except one, which is $2 \mu \mathrm{m}$ in (F). Color images available online at www.liebertpub .com/tea
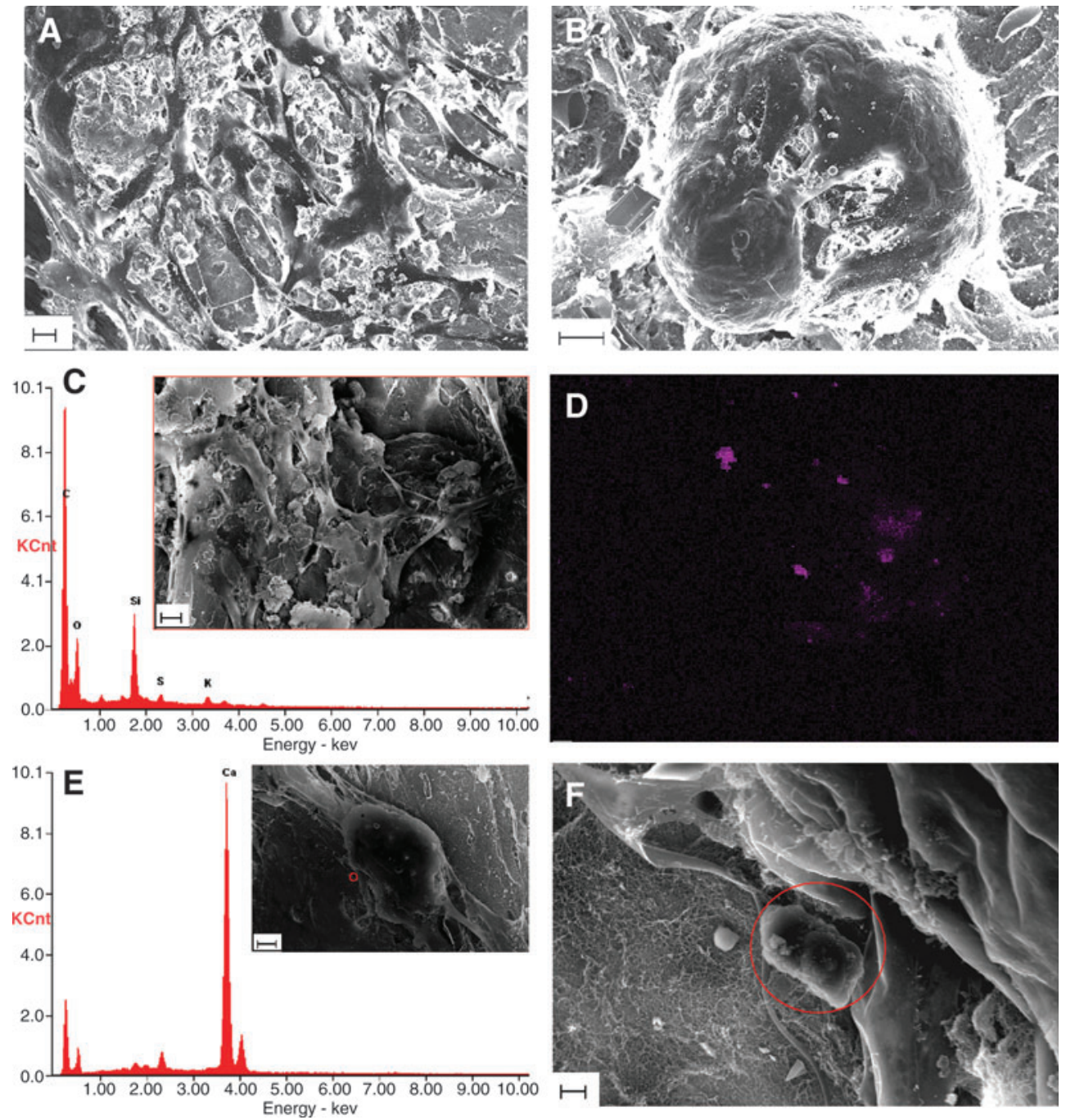

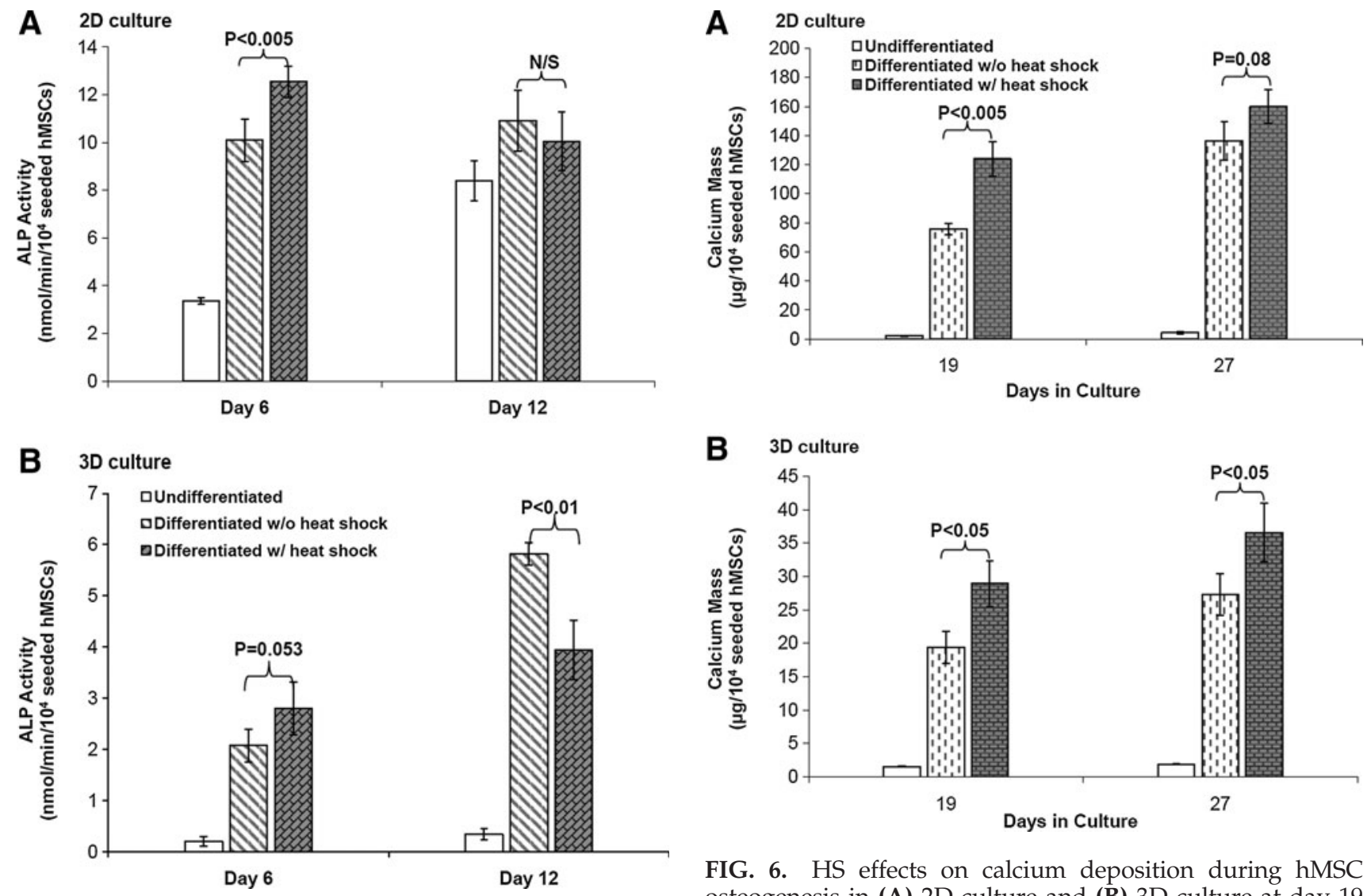

FIG. 5. Heat shock (HS) effects on alkaline phosphatase (ALP) activity measured by quantitative ALP assay in (A) 2D and (B) 3D culture at days 6 and 12 during osteogenesis.

than normal. ALP expression is a dynamic process and its activity usually peaks between day 9 and 12 during osteogenesis, varying from donor to donor. ${ }^{39}$ The decrease of ALP activities in 2D and 3D cultures on day 12 may be an indicator of the shifting of the ALP peak time. HS also induced an earlier mineralization in differentiated hMSCs and further enhanced the maturation of osteoblasts in the late stage. This earlier maturation may have significance for bone regeneration in vivo, as it will shorten the waiting time required to obtain differentiated and mature osteoblasts from hMSCs, which normally takes about 30 days for in vitro differentiation, for clinical bone repair using stem cell therapy.

Effects of mild hyperthermia on osteogenic gene expression during hMSC differentiation were not well studied. Cbfa1/Runx2 is an essential transcription factor for osteoblastic differentiation. ${ }^{42}$ OSX is a downstream gene of Run $x 2{ }^{43}$ and a necessary transcription factor for OP expression. ${ }^{42,44} \mathrm{OP}$ is an ECM protein that mediates cell attachment in osteoprogenitor cells and osteocytes, ${ }^{45}$ and considered as a late bone marker for osteoblast differentiation and mineralization. ${ }^{42}$ BMP2 plays an important role in embryonic bone development. ${ }^{46}$ We observed that HS dynamically upregulated these osteogenic genes in a timedependent manner during about 4 weeks of hMSC osteogenesis. In 2D hard surface culture, it seems that HS could upregulate late osteogenic genes much faster than the early ones (Fig. 7), demonstrated by upregulation of the OSX gene

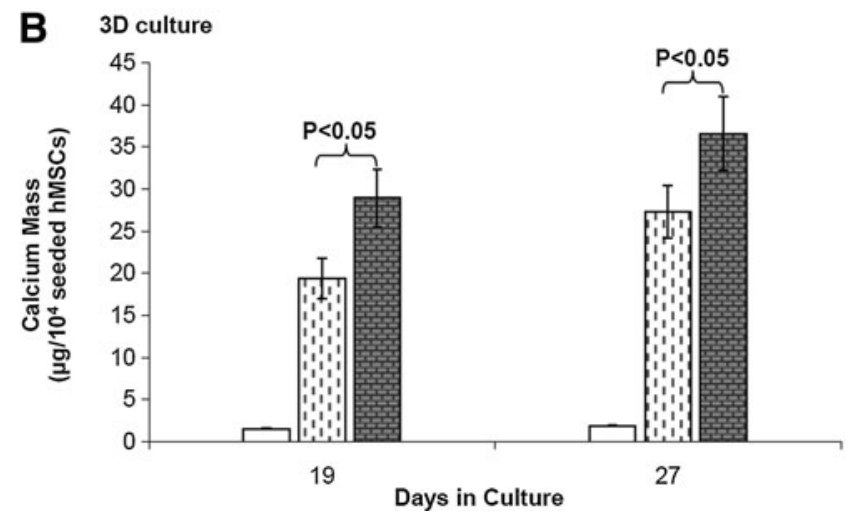

FIG. 6. HS effects on calcium deposition during hMSC osteogenesis in (A) 2D culture and (B) 3D culture at day 19 and 27.

at an early stage (day 11), $O P$ at the intermediate stage (day 19), but BMP2 and Runx2 at the late stage (day 25) only. Considering the gene expression pattern under HS, upregulating of late osteogenic genes and depressing the early ones during early to intermediate osteogenic differentiation stages, together with our data of enhanced early ALP activities and calcium deposition, we can speculate that HS may bypass some early osteo-specific genes, but turn on a few late osteo-specific genes directly. Since the basal production of ECM is likely regulated by Runx ${ }^{47}$ and BMP2 expression may be required for terminal differentiation into osteocytes, the upregulation of these two genes by HS in the late stage of hMSC osteogenesis is consistent with the results of enhanced mineralization in differentiated hMSC culture under thermal stimulation.

In 3D PuraMatrix culture, the up/downregulation patterns of four genes (i.e., BMP2, Runx2, OP, and OSX) comparing between osteogenic and osteogenic \& heat-shock conditions were similar to that of $2 \mathrm{D}$ culture. Interestingly, $B M P 2, O P$, and $O S X$ were not upregulated in differentiated hMSCs comparing to the control (Fig. 8) despite the definite hMSC differentiation confirmed by cell morphology (Fig. 2), ALP activities (Fig. 5B), and calcium deposition (Fig. 6B). Other osteo-specific genes must be turned on in this case. DNA microarray technology could be used to identify those genes. On the other hand, the lower expression of four osteogenic genes in 3D soft PuraMatrix culture are consistent with the delay of differentiation reflected by lower ALP activities or calcium deposition in 3D culture compared with 

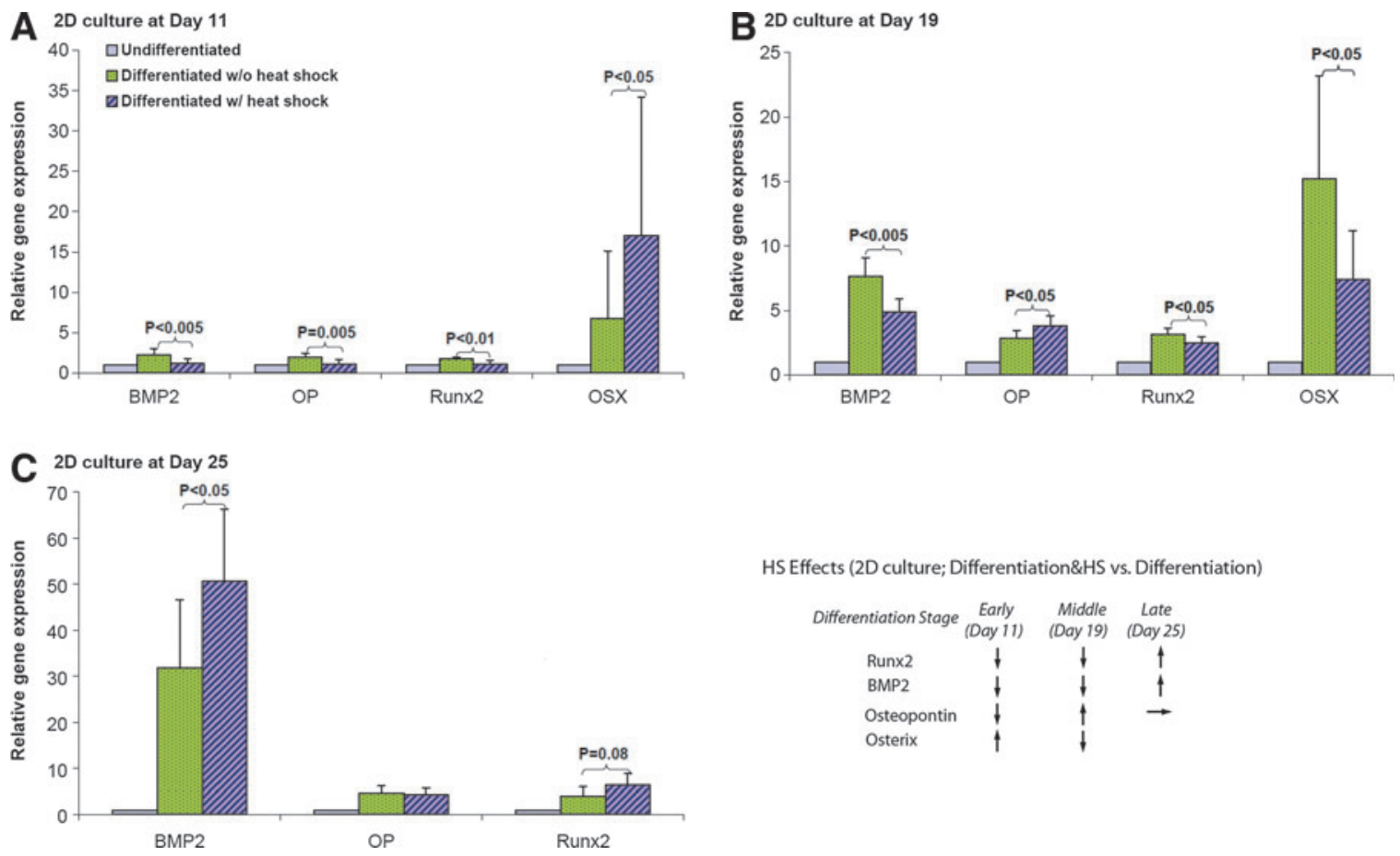

HS Effects (2D culture; Differentiation\&HS vs. Differentiation)

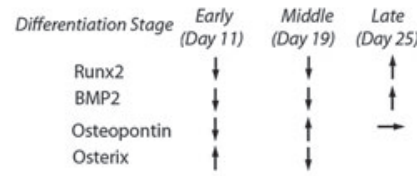

FIG. 7. Gene expression of osteo-specific markers (bone morphogenetic protein 2 [BMP2], osteopontin [OP], runt-related transcription factor 2 [Runx2], OSX) assessed by real-time reverse transcription-polymerase chain reaction (RT-PCR) in 2D culture at (A) day 11 (B) day 19 (C) day 25 after differentiation. Comparison was between differentiated hMSCs with and without the effect of HS. Undifferentiated hMSCs were used as control (mean \pm standard deviation [SD], $n=6-16$ ). $p$-Values were calculated using Student's t-test. Color images available online at www.liebertpub.com/tea

2D (Figs. 5 and 6). The hard polystyrene surface of 2D culture plates (Young's modulus of $3.5 \mathrm{GPa}$ ) is much stiffer than the soft substrate, $0.25 \%$ PuraMatrix (storage modulus of $2.5 \mathrm{kPa}$ at $0.5 \%$ ). In general, matrix elasticity, or the stiffness of the
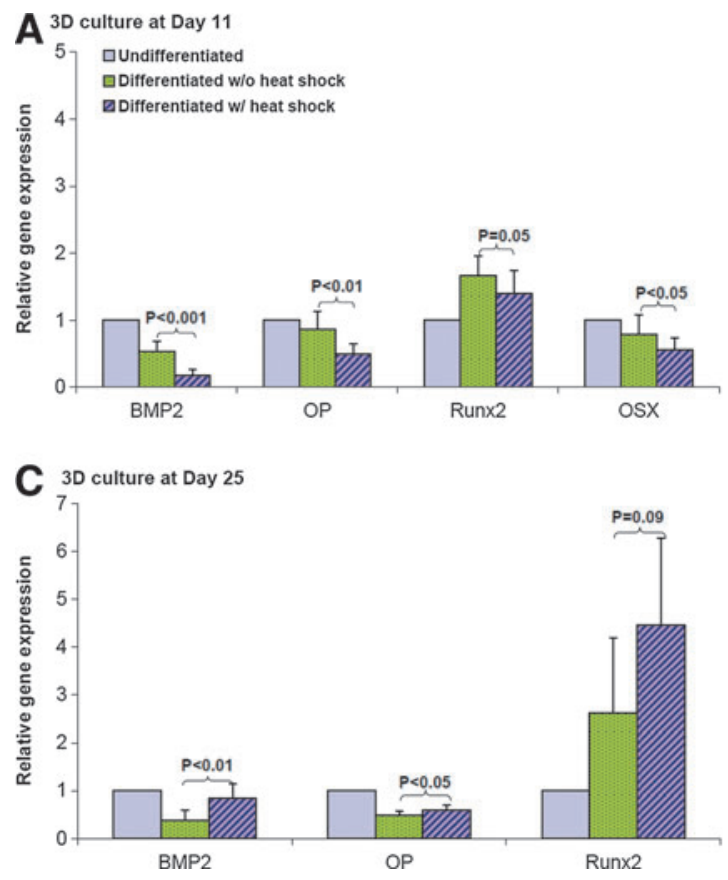

substrate could significantly affect the stem cell differentiation directions. ${ }^{48}$ Apparently, the 3D soft hydrogel seemed to delay the hMSC osteogenesis compared to tissue culture plates, while the hard surface of $2 \mathrm{D}$ culture plate itself

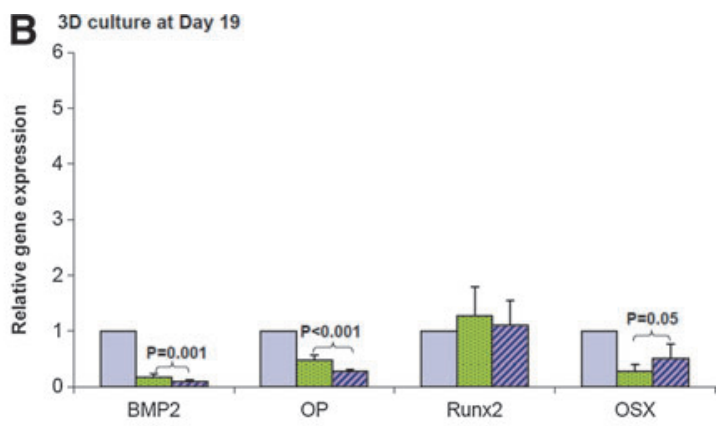

HS Effects (3D culture; Differentiation\&HS vs. Differentiation)

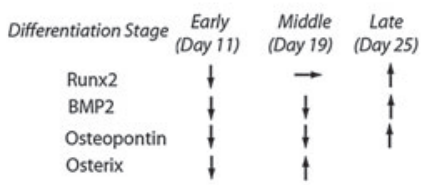

FIG. 8. Gene expression of osteo-specific markers (BMP2, OP, Runx2, OSX) assessed by real-time RT-PCR in 3D culture at (A) day 11 (B) day 19 (C) day 25 during differentiation. Comparison was between differentiated hMSCs with and without the effect of HS. Undifferentiated hMSCs were used as control (mean \pm SD, $n=6-12$ ). P values were calculated using Student's $t$ test. Color images available online at www.liebertpub.com/tea 
Table 2. Expression of Heat Shock Proteins (HSP27, HSC70 + HSP70, HSP90) on Day 4, 11, 18, and 25 During Differentiation in Human Mesenchymal Stem Cell Cultures

\begin{tabular}{|c|c|c|c|c|c|}
\hline HSP/actin Days & & Day 4 & Day 11 & Day 18 & Day 25 \\
\hline \multicolumn{6}{|l|}{$2 \mathrm{D}$} \\
\hline HSP27 & $\begin{array}{l}\mathrm{C} \\
\mathrm{O} \\
\mathrm{H}\end{array}$ & $\begin{array}{l}1.339 \pm 0.107 \\
1.554 \pm 0.069^{\mathrm{a}} \\
1.703 \pm 0.105\end{array}$ & $\begin{array}{l}1.690 \pm 0.137 \\
2.037 \pm 0.099^{\mathrm{a}} \\
2.094 \pm 0.066\end{array}$ & $\begin{array}{l}1.244 \pm 0.109 \\
3.168 \pm 0.385^{\mathrm{a}} \\
3.412 \pm 0.545\end{array}$ & $\begin{array}{l}1.211 \pm 0.037 \\
1.378 \pm 0.071^{\mathrm{a}} \\
1.555 \pm 0.133\end{array}$ \\
\hline HSC70 + HSP70 & $\begin{array}{l}\mathrm{C} \\
\mathrm{O} \\
\mathrm{H}\end{array}$ & $\begin{array}{l}0.126 \pm 0.002 \\
0.097 \pm 0.008^{\mathrm{a}} \\
0.196 \pm 0.005^{\mathrm{b}}\end{array}$ & $\begin{array}{l}0.224 \pm 0.040 \\
0.113 \pm 0.035^{\mathrm{a}} \\
0.297 \pm 0.052^{\mathrm{b}}\end{array}$ & $\begin{array}{l}0.201 \pm 0.005 \\
0.075 \pm 0.017^{\mathrm{a}} \\
0.279 \pm 0.022^{\mathrm{b}}\end{array}$ & $\begin{array}{l}0.297 \pm 0.015 \\
0.129 \pm 0.022^{\mathrm{a}} \\
0.322 \pm 0.067^{\mathrm{b}}\end{array}$ \\
\hline HSP90 & $\begin{array}{l}\mathrm{C} \\
\mathrm{O} \\
\mathrm{H}\end{array}$ & $\begin{array}{l}0.024 \pm 0.006 \\
0.063 \pm 0.019^{\mathrm{a}} \\
0.028 \pm 0.006^{\mathrm{b}}\end{array}$ & $\begin{array}{l}0.083 \pm 0.017 \\
0.102 \pm 0.016 \\
0.098 \pm 0.012\end{array}$ & $\begin{array}{l}0.046 \pm 0.004 \\
0.055 \pm 0.010 \\
0.048 \pm 0.016\end{array}$ & $\begin{array}{l}0.078 \pm 0.030 \\
0.069 \pm 0.017 \\
0.090 \pm 0.017\end{array}$ \\
\hline \multicolumn{6}{|l|}{$3 \mathrm{D}$} \\
\hline HSP27 & $\begin{array}{l}\mathrm{C} \\
\mathrm{O} \\
\mathrm{H}\end{array}$ & $\begin{array}{l}\text { ND } \\
\text { ND } \\
\text { ND }\end{array}$ & $\begin{array}{l}0.064 \pm 0.018 \\
0.137 \pm 0.024^{\mathrm{a}} \\
0.159 \pm 0.021\end{array}$ & $\begin{array}{l}0.054 \pm 0.017 \\
0.092 \pm 0.018^{\mathrm{a}} \\
0.100 \pm 0.025\end{array}$ & $\begin{array}{l}0.070 \pm 0.022 \\
0.199 \pm 0.022^{\mathrm{a}} \\
0.206 \pm 0.058\end{array}$ \\
\hline
\end{tabular}

$n=3$, mean \pm standard deviation.

${ }^{\mathrm{a}} p<0.05\left[\mathrm{O}\right.$ vs. C], ${ }^{\mathrm{b}} p<0.05[\mathrm{O}$ vs. H]

2D, two-dimensional; 3D, three-dimensional; HS, heat shock; C, growth; $\mathrm{O}$, osteogenic; $\mathrm{H}$, osteogenic \& heat shocked; ND, not detected.

actually facilitates osteogenesis. ${ }^{48}$ This may be one of reasons that the heat-induced higher calcium deposition was still very significant in 3D culture even on day 27 , but not very significant in $2 \mathrm{D}$ culture (Fig. 6). HS may have forced
hMSCs in the osteogenic medium on soft hydrogel more toward osteo-lineage. In addition, the 3D culture environment enables intercellular contacts from different layers. Whether or not the 3D culture configuration itself
A Ctri2D OS2D HS2D
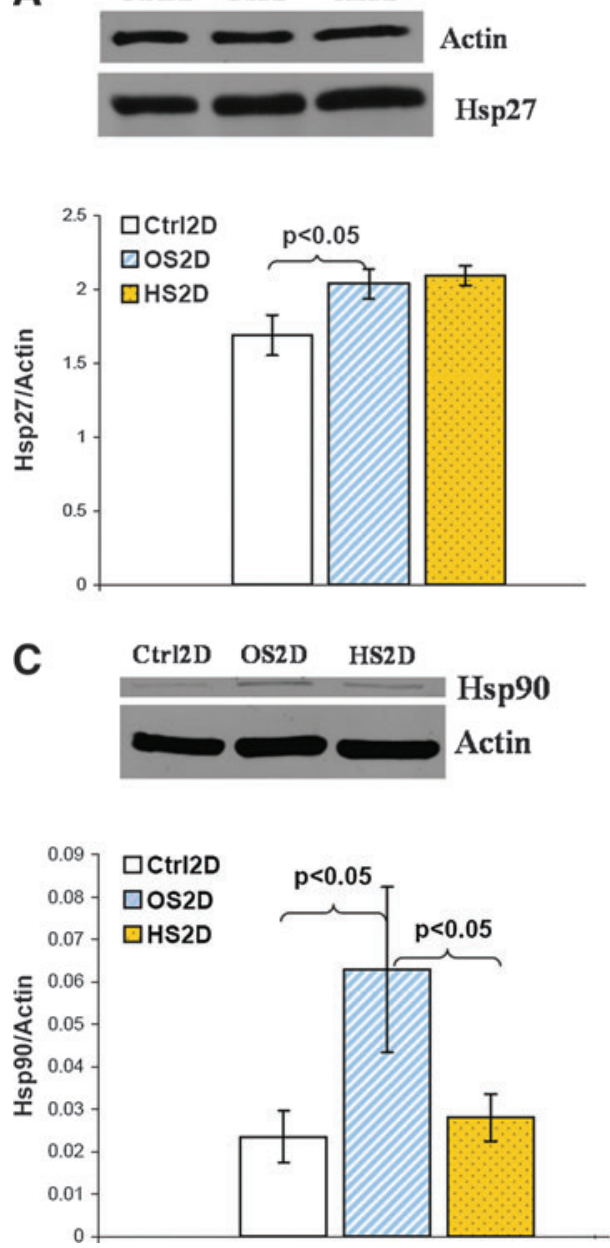

B

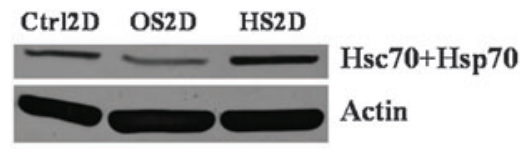

FIG. 9. The representative Western blot membrane followed by a corresponding bar graph of (A) HSP27 expression in 2D culture on day 11 during differentiation, (B) $\mathrm{HSC70}+\mathrm{HSP70}$ expression in 2D culture on day 11 during differentiation, (C) HSP90 expression in 2D culture on day 4 during differentiation and (D) HSP27 expression in 3D culture on day 11 during differentiation. (Ctrl: undifferentiated hMSCs, OS: differentiated hMSCs, and HS: differentiated hMSCs with HS). Color images available online at www.liebertpub .com/tea

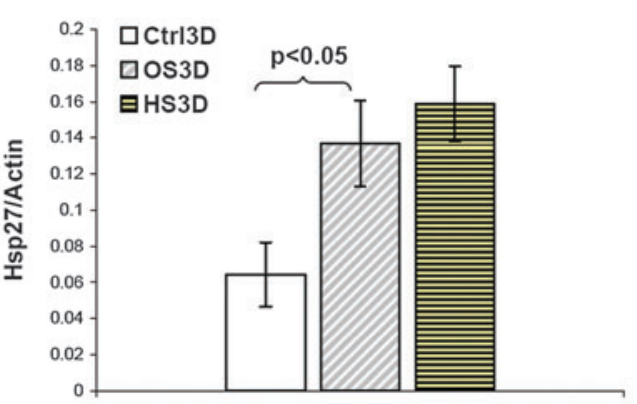



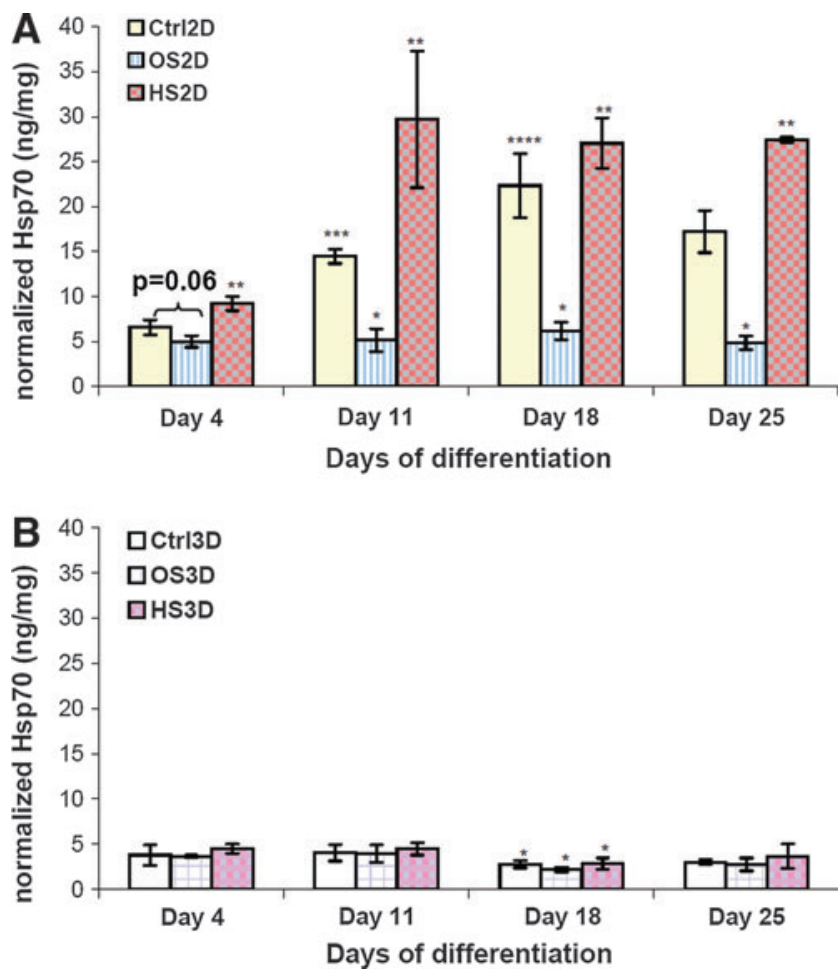

FIG. 10. Inducible HSP70 expression measured by ELISA in (A) 2D culture and (B) 3D culture on days 4, 11, 18, and 25 during differentiation. (Ctrl: undifferentiated hMSCs, OS: differentiated hMSCs without HS, and HS: differentiated hMSCs with HS). In panel (A), ${ }^{*} p<0.05$ (OS culture compared to the corresponding Ctrl culture on the same day), ${ }^{* *} p<$ 0.05(HS culture compared to the corresponding OS culture on the same day), ${ }^{* * *} p<0.05$ (day 11 Ctrl culture compared to day 4 Ctrl culture), ${ }^{* * * *} p<0.05$ (day 18 Ctrl culture compared to day 11 Ctrl culture). In panel (B), ${ }^{*} p<0.05$ (The corresponding conditions (Ctrl, OS, HS) compared between day 18 and day 11). Color images available online at www.liebertpub.com/tea

triggers the different differentiation signals needs to be investigated more.

To further study the mechanisms in the HS-enhanced osteogenic differentiation of hMSCs, HSP expression, including HSP27, HSC70 + HSP70, HSP70 alone, and HSP90, was investigated. HSPs are chaperon proteins, which are upregulated in response to stresses. HSP27 is a multifunctional protein with a molecular weight of $27 \mathrm{kDa}$. It is an important regulator in almost all cellular processes, such as migration, proliferation, differentiation, and apoptosis due to its close association with actin. HSP27 overexpression during different stages of cell differentiation and development was reported. ${ }^{49}$ In this study, the significantly increased HSP27 expression in differentiated cells (Fig. 9A, D) may be due to cytoskeleton reorganization during differentiation, resulting in the morphological changes of differentiated cells. Despite this, exposure of differentiated hMSCs to HS did not further stimulate the expression of HSP27, which is consistent with no observable morphological differences between heated \& differentiated hMSCs and nonheated ones. However, the subtle change of phosphorylated HSP27 needs to be studied in the future.

HSP70 (MW: $70 \mathrm{kDa}$ ) have two types, constitutive HSC70 and inducible HSP70. ${ }^{50}$ HSP90 also has two isoforms, inducible form HSP90 $\alpha$ and constitutive form HSP90 $\beta$, and the level of expression is usually lower in the former than the latter. Both HSC70/HSP70 and HSP90 are involved in various cell processes, such as proliferation, differentiation, and apoptosis, ${ }^{50,51}$ by correcting misfolded proteins under stresses or helping to fold newly synthesized proteins during normal physiological conditions. The reduced expression of HSP70 in differentiated hMSCs (Fig. 9B) may be a result of extremely low cell proliferation during differentiation. Mild hyperthermia increased biochemical reaction rates in hMSCs, which induced overexpression of HSC70 and HSP70 in differentiated plus heat shocked hMSCs. The elevated HSP70 expression was also reported in a previous study using hMSC 2D culture and hyperthermia. ${ }^{21}$ In contrast, HSP90 was upregulated in differentiated cells compared to undifferentiated cells, and HS downregulated its expression (Fig. 9C), but significance was only seen in the early stage of differentiation. The mechanisms remained unclear, but studies on different lineage also confirmed the importance of HSP90 $\beta$ overexpression from early to late stage of germ cell differentiation and also for early embryonic development. ${ }^{52,53}$ Both HSP70 and 90 are molecular chaperones, but it seems that they modulate cell differentiation through their own and maybe different signaling pathways. There was no similar study reporting the HSP90 expression in differentiated hMSCs toward bone lineage with HS. The underlying mechanisms of its downregulation remain unclear. The upregulation of HSP90 may not be a must-need to sustain an enhanced differentiation due to the fact that HSP70 is upregulated. Further experiments will be required to find out what specific roles it plays. In 3D PuraMatrix culture, the expression of three HSPs was significantly lower, which may be correlated with the less stresses cells experienced on the peptide hydrogel during growth or differentiation.

In summary, this is the first comprehensive study of HS effects on hMSC differentiation into osteoblasts in a 3D hydrogel scaffold. Our results showed that periodically mild HS was able to facilitate MSC differentiation into osteoblasts and enhance their maturity. In addition, this study provided a 3D MSC culture model in vitro to mimic an in vivo environment surrounding bone marrow. Altogether, our data may have a significant impact on the development of a therapeutic thermal treatment protocol to enhance stem cell differentiation during cell transplantation. For bone regeneration in vivo, differentiated osteoblasts from hMSCs can be transplanted into bone defects, by injection using PuraMatrix as a carrying medium coupled with a heating device developed according to the results of this study. Future studies will use siRNAs/shRNAs targeting HSP70 and HSP90 to investigate the role of HSPs during hMSC differentiation with or without HS. Other heating patterns and temperatures (e.g., $39^{\circ} \mathrm{C}$, known as the elevated body temperature during exercise ${ }^{38}$ ) will be evaluated in further studies to optimize the heating protocol for hMSC differentiation. Other biomaterials with different stiffness for bone and cartilage regeneration will be evaluated in the future using the similar experimental protocol described in this study. Given the fact that OA greatly affects the elderly people, and it requires regeneration of both bone and cartilage at late stage, hyperthermia (via ultrasound and microwaves etc.) may represent a less invasive and simple therapy for clinical joint repair, and thus potentially be a promising approach in bone and cartilage regenerative medicine. 


\section{Acknowledgments}

We would like to thank the kindness of Dr. Jeremy Mao and his lab at Columbia University to introduce us the hMSC isolation technologies. This project is funded by NIH/NIA SC2AG036823 and the NYSTEM program from New York State Health Department.

\section{Disclosure Statement}

No competing financial interests exist.

\section{References}

1. Kraus, V.B. Pathogenesis and treatment of osteoarthritis. Med Clin N Am 81, 85, 1997.

2. Marion, N.W., and Mao, J.J. Mesenchymal stem cells and tissue engineering. In: Lanza, R., and Klimanskaya, I., eds. Stem Cell Tools and Other Experimental Protocols. San Diego: Elsevier Academic Press, Inc., 2006, pp. 339-361.

3. Shen FH, Z.Q., Lv, Q., Choi, L., Balian, G., Li, X., and Laurencin, C.T. Osteogenic differentiation of adipose-derived stromal cells treated with GDF- 5 cultured on a novel threedimensional sintered microsphere matrix. Spine J 6, 615, 2006.

4. Misawa, H., Kobayashi, N., Soto-Gutierrez, A., Chen, Y., Yoshida, A., Rivas-Carrillo, J.D., et al. PuraMatrix (TM) facilitates bone regeneration in bone defects of calvaria in mice. Cell Transplant 15, 2006.

5. Mao, J.J. Stem-cell-driven regeneration of synovial joints. Biol Cell 97, 2005.

6. Shih, Y.R.V., Chen, C.N., Tsai, S.W., Wang, Y.J., and Lee, O.K. Growth of mesenchymal stem cells on electrospun type I collagen nanofibers. Stem Cells 24, 2006.

7. Hamada, K., Hirose, M., Yamashita, T., and Ohgushil, H. Spatial distribution of mineralized bone matrix produced by marrow mesenchymal stem cells in self-assembling peptide hydrogel scaffold. J Biomed Mater Res Part A 84A, 2008.

8. Kim, H.K., Shim, W.S., Kim, S.E., Lee, K.H., Kang, E., Kim, J.H., et al. Injectable in situ-forming $\mathrm{pH} /$ thermo-sensitive hydrogel for bone tissue engineering. Tissue Eng Part A 15, 2009.

9. Moreau, J.L., and Xu, H.H.K. Mesenchymal stem cell proliferation and differentiation on an injectable calcium phosphate-chitosan composite scaffold. Biomaterials 30, 2009.

10. Chun, C., Lim, H.J., Hong, K.Y., Park, K.H., and Song, S.C. The use of injectable, thermosensitive poly(organophosphazene)RGD conjugates for the enhancement of mesenchymal stem cell osteogenic differentiation. Biomaterials 30, 2009.

11. Alhadlaq, A., and Mao, J.J. Mesenchymal stem cells: isolation and therapeutics. Stem Cells Dev 13, 2004.

12. Kratchmarova, I., Blagoev, B., Haack-Sorensen, M., Kassem, M., and Mann, M. Mechanism of divergent growth factor effects in mesenchymal stem cell differentiation. Science 308, 2005.

13. Hughes, F.J., Turner, W., Belibasakis, G., and Martuscelli, G. Effects of growth factors and cytokines on osteoblast differentiation. Periodontol 2000 41, 2006.

14. David, V., Martin, A., Lafage-Proust, M.H., Malaval, L., Peyroche, S., Jones, D.B., et al. Mechanical loading downregulates peroxisome proliferator-activated receptor gamma in bone marrow stromal cells and favors osteoblastogenesis at the expense of adipogenesis. Endocrinology 148, 2007.

15. Ogawa, H. [Effects of the localized thermal enhancement on new bone formation following mechanical expansion of the rat sagittal suture]. Nippon Kyosei Shika Gakkai Zasshi 49, 1990.
16. Brookes, M., Richards, D.J., and Singh, M. Vascular sequelae of experimental osteotomy. Angiology 21, 1970.

17. Takahashi, K.A., Tonomura, H., Arai, Y., Terauchi, R., Honjo, K., Hiraoka, N., et al. Hyperthermia for the treatment of articular cartilage with osteoarthritis. Int J Hyperthermia 25, 2009.

18. Doyle, J.R., and Smart, B.W. Stimulation of bone growth by short-wave diathermy. J Bone Joint Surg-Am Vol 45, 1963.

19. Richards, V., and Stofer, R. The stimulation of bone growth by internal heating. Surgery 46, 1959.

20. Brodin, H. Longitudinal bone growth, the nutrition of the epiphyseal cartilages and the local blood supply; an experimental study in the rabbit. Acta Orthop Scand Suppl 20, 1955.

21. Shui C, S.A. Mild heat shock induces proliferation, alkaline phosphatase activity, and mineralization in human bone marrow stromal cells and Mg-63 cells in vitro. J Bone Miner Res 16, 731, 2001.

22. Ye, C.P., Heng, B.C., Liu, H., Toh, W.S., and Cao, T. Culture media conditioned by heat-shocked osteoblasts enhances the osteogenesis of bone marrow-derived mesenchymal stromal cells. Cell Biochem Funct 25, 2007.

23. Nørgaard, R., Kassem, M., Rattan, S.I. Heat shock-induced enhancement of osteoblastic differentiation of hTERTimmortalized mesenchymal stem cells. Ann N Y Acad Sci 1067, 443, 2006.

24. Trieb, K., Blahovec, H., and Kubista, B. Effects of hyperthermia on heat shock protein expression, alkaline phosphatase activity and proliferation in human osteosarcoma cells. Cell Biochem Funct 25, 2007.

25. Genove, E., Shen, C., Zhang, S.G., and Semino, C.E. The effect of functionalized self-assembling peptide scaffolds on human aortic endothelial cell function. Biomaterials 26, 2005.

26. Elastic properties and Young's modulus for some materials. www.engineeringtoolbox.com/young-modulus-d_417.html

27. Winer, J.P., Janmey, P.A., McCormick, M.E., and Funaki, M. Bone marrow-derived human mesenchymal stem cells become quiescent on soft substrates but remain responsive to chemical or mechanical stimuli. Tissue Eng Part A 15, 2009.

28. Zhang, S.G., Holmes, T., Lockshin, C., and Rich, A. Spontaneous assembly of a self-complementary oligopeptide to form a stable macroscopic membrane. Proc Natl Acad Sci U S A 90, 1993.

29. Zhang, S.G., Holmes, T.C., Dipersio, C.M., Hynes, R.O., Su, X., and Rich, A. Self-complementary oligopeptide matrices support mammalian-cell attachment. Biomaterials 16, 1995.

30. Semino, C.E., Kasahara, J., Hayashi, Y., and Zhang, S.G. Entrapment of migrating hippocampal neural cells in threedimensional peptide nanofiber scaffold. Tissue Eng 10, 2004.

31. Wang, S., Nagrath, D., Chen, P.C., Berthiaume, F., and Yarmush, M.L. Three-dimensional primary hepatocyte culture in synthetic self-assembling peptide hydrogel. Tissue Eng Part A 14, 2008.

32. Semino, C.E., Merok, J.R., Crane, G.G., Panagiotakos, G., and Zhang, S.G. Functional differentiation of hepatocytelike spheroid structures from putative liver progenitor cells in three-dimensional peptide scaffolds. Differentiation 71, 2003.

33. Kisiday, J., Jin, M., Kurz, B., Hung, H., Semino, C., Zhang, S., et al. Self-assembling peptide hydrogel fosters chondrocyte extracellular matrix production and cell division: implications for cartilage tissue repair. Proc Natl Acad Sci U S A 99, 2002.

34. Bokhari, M.A., Akay, G., Zhang, S.G., and Birch, M.A. Enhancement of osteoblast growth and differentiation in vitro on a peptide hydrogel-polyHIPE polymer hybrid material. Biomaterials 26, 2005. 
35. Dickhut, A., Gottwald, E., Steck, E., Heisel, C., and Richter, W. Chondrogenesis of mesenchymal stem cells in gel-like biomaterials in vitro and in vivo. Front Biosci 13, 2008.

36. Erickson, I.E., Huang, A.H., Chung, C., Li, R.T., Burdick, J.A., and Mauck, R.L. Differential maturation and structurefunction relationships in mesenchymal stem cell- and chondrocyte-seeded hydrogels. Tissue Eng Part A 15, 2009.

37. Yoshimi, R., Yamada, Y., Ito, K., Nakamura, S., Abe, A., Nagasaka, T., et al. Self-assembling peptide nanofiber scaffolds, platelet-rich plasma, and mesenchymal stem cells for injectable bone regeneration with tissue engineering. J Craniofac Surg 20, 2009.

38. Lim, C.L., Byrne, C., and Lee, J.K.W. Human thermoregulation and measurement of body temperature in exercise and clinical settings. Ann Acad Med Singap 37, 2008.

39. Jaiswal, N., Haynesworth, S.E., Caplan, A.I., and Bruder, S.P. Osteogenic differentiation of purified, culture-expanded human mesenchymal stem cells in vitro. J Cell Biochem 64, 1997.

40. Goldstein, J., Newbury, D., Joy, D., Lyman, C., Echlin, P., Lifshin, et al. Scanning Electron Microscopy and X-Ray Microanalysis, third edition. New York: Springer, 2003.

41. Hee, C.K., Jonikas, M.A., and Nicoll, S.B. Influence of threedimensional scaffold on the expression of osteogenic differentiation markers by human dermal fibroblasts. Biomaterials 27, 2006.

42. Hirata, A., Sugahara, T., and Nakamura, H. Localization of Runx2, osterix, and osteopontin in tooth root formation in rat molars. J Histochem Cytochem 57, 2009.

43. Johnsen, S.A., Subramaniam, M., Ruesink, T.J., and Spelsberg, T.C. Opposing roles of osterix and TGF beta inducible early gene in osteoblast differentiation downstream of Cbfa1/Runx2. J Bone Miner Res 17, 2002.

44. Cao, Y., Zhou, Z.C., de Crombrugghe, B., Nakashima, K., Guan, H., Duan, X.P., et al. Osterix, a transcription factor for osteoblast differentiation, mediates antitumor activity in murine osteosarcoma. Cancer Res 65, 2005.

45. Nakamura, H., Kenmotsu, S., Sakai, H., and Ozawa, H. Localization of $\mathrm{CD} 44$, the hyaluronate receptor, on the plasma-membrane of osteocytes and osteoclasts in rat tibiae. Cell Tissue Res 280, 1995.
46. Friedman, M.S., Long, M.W., and Hankenson, K.D. Osteogenic differentiation of human mesenchymal stem cells is regulated by bone morphogenetic protein- 6 . J Cell Biochem 98, 2006.

47. Kern, B., Shen, J.H., Starbuck, M., and Karsenty, G. Cbfa1 contributes to the osteoblast-specific expression of type I collagen genes. J Biol Chem 276, 2001.

48. Even-Ram, S., Artym, V., and Yamada, K.M. Matrix control of stem cell fate. Cell 126, 2006.

49. Kostenko, S., and Moens, U. Heat shock protein 27 phosphorylation: kinases, phosphatases, functions and pathology. Cell Mol Life Sci 66, 2009.

50. Turturici, G., Geraci, F., Candela, M.E., Giudice, G., Gonzalez, F., and Sconzo, G. Hsp70 localizes differently from chaperone Hsc70 in mouse mesoangioblasts under physiological growth conditions. J Mol Histol 39, 2008.

51. Sreedhar, A.S., Kalmar, E., Csermely, P., and Shen, Y.F. Hsp90 isoforms: functions, expression and clinical importance. FEBS Lett 562, 2004.

52. Hilscher, B., and Hilscher, W. Kinetics of gametogenesis .2. Comparative autoradiographic studies of oogonia and multiplying prospermatogonia of wistar rat. Cell Tissue Res 190, 1978.

53. Vanmuylder, N., Werry-Huet, A., Rooze, M., and Louryan, S. Heat shock protein HSP86 expression during mouse embryo development, especially in the germ-line. Anat Embryol 205, 2002.

Address correspondence to: Sihong Wang, PhD Department of Biomedical Engineering

The City College of New York Steinman Hall, Room 434 160 Convent Ave. New York, NY, 10031

E-mail: shwang@ccny.cuny.edu

Received: February 4, 2012 Accepted: October 3, 2012

Online Publication Date: November 26, 2012 